\title{
ADENOCARCINOMA CON DIFERENCIACIÓN APOCRINA DE LA GLÁNDULA LAGRIMAL
}

\section{ADENOCARCINOMA WITH APOCRINE DIFFERENTIATION OF THE LACRIMAL GLAND}

\author{
LOZANO $\mathrm{M}^{1}$, GONZALEZ F ${ }^{2}$
}

\section{RESUMEN}

Caso clínico: Se presenta el caso de un varón de 74 años con una tumoración orbitaria localizada en la fosa lagrimal izquierda. La exploración oftalmológica mostró hipotropía, exoftalmos y ptosis palpebral del ojo izquierdo.

Discusión: La tumoración se extirpó mediante una orbitotomía lateral y el resultado anatomopatológico fue de adenocarcinoma de la glándula lagrimal con diferenciación apocrina.

Palabras clave: Adenocarcinoma, glándula lagrimal, orbita, apocrina, fosa lagrimal.

\begin{abstract}
Case report: A 74-year-old male presented with an orbital tumor located in the left lacrimal fossa. Ocular examination showed hypotropia, exophthalmos and palpebral ptosis.

Discussion: Removal of the tumor was performed by a lateral orbitotomy. The histopathologic study showed a tumor consistent with an adenocarcinoma of the lacrimal gland with apocrine differentiation (Arch Soc Esp Oftalmol 2007; 82: 229-232).
\end{abstract}

Key words: Adenocarcinoma, lacrimal gland, orbit, apocrine, lacrimal fossa.

\section{INTRODUCCIÓN}

Los tumores originados en la glándula lagrimal representan aproximadamente el $10 \%$ de los procesos tumorales orbitarios $(1,2)$ y tienen una gran diversidad de tipos anatomopatológicos (3). Las neoplasias con diferenciación aprocina se presentan raramente en la región ocular (4) y todavía menos frecuentemente en la glándula lagrimal (4) que es una glándula ecrina. A continuación se describe un caso de adenocarcinoma de glándula lagrimal con diferenciación apocrina.

\footnotetext{
Recibido: 17/3/06. Aceptado: 23/3/07. Medicina de la Universidad de Santiago de Compostela. España.

1 Licenciado en Medicina.

2 Doctor en Medicina.

Correspondencia:

Francisco Gonzalez

Departamento de Fisiología, Facultad de Medicina

Universidad de Santiago de Compostela

C/. San Francisco

15782 Santiago de Compostela

España

E-mail: francisco.gonzalez@usc.es
}

Servicio de Oftalmología del Complejo Hospitalario Universitario de Santiago de Compostela. España. Departamento de Fisiología. Facultad de

Este trabajo ha sido posible gracias a las ayudas BFU2004-01839 del Ministerio de Educación y Ciencia y FEDER. 


\section{CASO CLÍNICO}

Varón de 74 años de edad que es remitido por presentar una tumoración en la fosa lagrimal izquierda. El hallazgo fue detectado casualmente al realizar una tomografía axial computarizada (TAC) para descartar un accidente cerebrovascular. A la exploración oftalmológica presentaba exoftalmos del ojo izquierdo (OI), ptosis palpebral e hipotropía (fig. 1), con limitación de los movimientos. La agudeza visual en ambos ojos era de contar dedos a dos metros, que se atribuyó en ese momento a su posible accidente cerebral vascular (ACV) previo. Refirió no haber tenido nunca dolor en la región orbitaria izquierda. La tumoración observada en la TAC (fig. 2) estaba localizada en la fosa lagrimal y tenía un tamaño aproximado de $2 \times 2 \mathrm{~cm}$, que contactaba el globo ocular sin producir compresión significativa y con el músculo recto externo sin aparente compromiso del mismo. Además había calcificaciones y soluciones de continuidad con bordes esclerosos en las paredes óseas superior y lateral de la orbita adyacentes a la lesión. Un estudio adicional realizado con resonancia magnética nuclear (RMN) mostró hallazgos similares (fig. 3).

Se indicó tratamiento quirúrgico y se accedió al tumor a través de una orbitotomía lateral, observándose una masa redondeada en la fosa lagrimal, de color blanquecino-grisáceo, pseudoencapsulada y de consistencia dura y firme (fig. 4) adherida al tejido circundante que erosionaba y perforaba el hueso adyacente. La exéresis fue completa y el resultado anatomopatológico fue de adenocarcinoma de la glándula lagrimal con diferenciación apocrina. Después de la cirugía el paciente fue tratado con antibióticos, corticoides orales, lubricantes corneales y

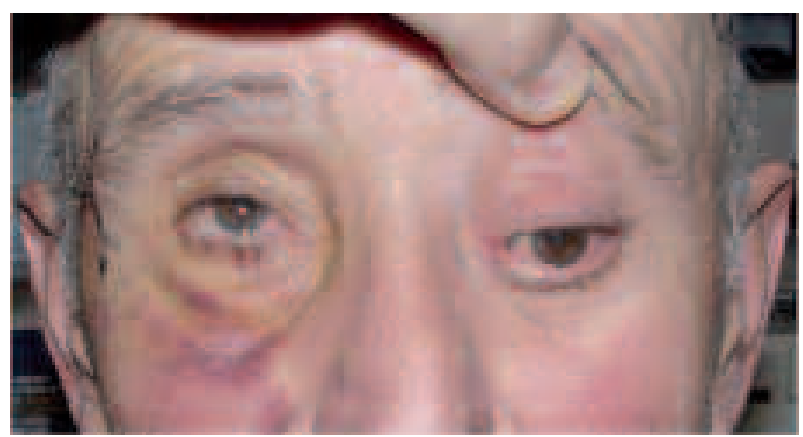

Fig. 1: Obsérvese la ptosis palpebral, el exoftalmos y la hipotropía ocular izquierda con limitación de los movimientos oculares.

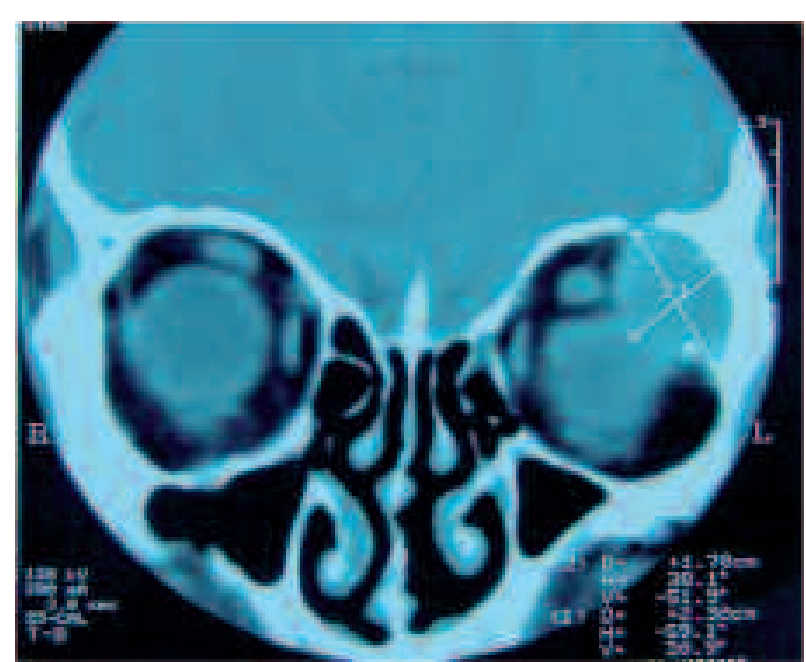

Fig. 2: Corte coronal de una tomografía axial computarizada mostrando una masa en la fosa lagrimal izquierda. Puede observarse una solución de continuidad ósea en el techo de la órbita (flecha blanca) y una calcificación en la periferia del tumor (flecha negra).

lágrimas artificiales. A los 24 días después de la cirugía apareció una úlcera corneal que requirió recubrimiento conjuntival para su resolución. Tras una consulta con el Servicio de Oncología, dado el estado general del paciente (enfermedad cerebrovascular, síndrome confunsional de naturaleza comicial), se decidió inicialmente la abstención de medidas terapéuticas o diagnósticas adicionales. El

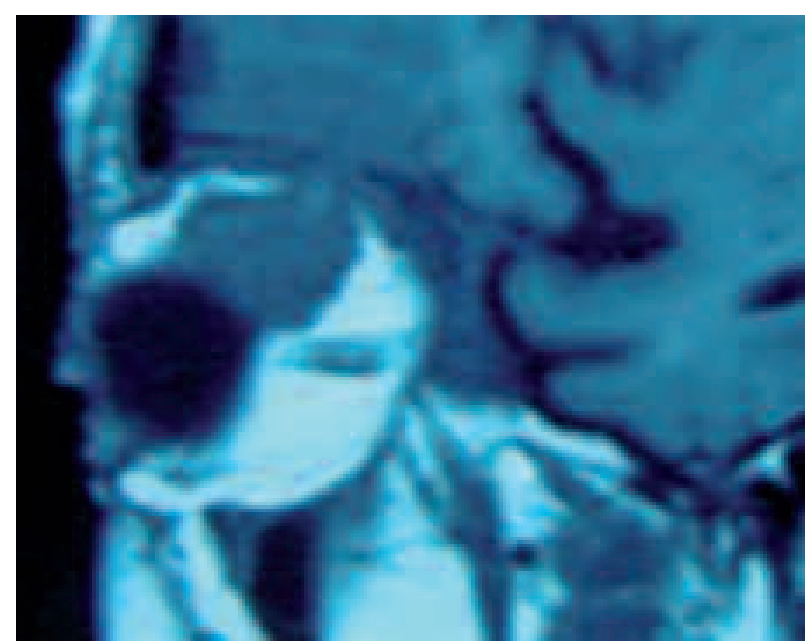

Fig. 3: Corte sagital de resonancia magnética nuclear mostrando la localización del tumor en la fosa lagrimal izquierda. Obsérvese la invasión del techo de la orbita (flecha blanca). 


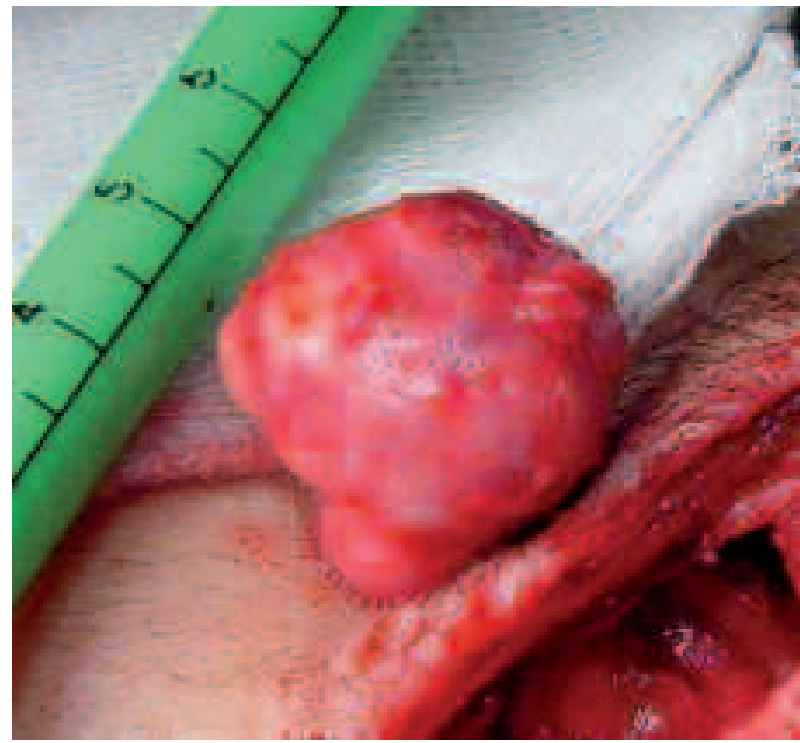

Fig. 4: Aspecto macroscópico del tumor tras ser extirpado mediante una orbitotomía lateral.

paciente falleció al cabo de un año de la extirpación del tumor a consecuencia de un nuevo ACV.

\section{DISCUSIÓN}

La edad media de presentación de los tumores de la glándula lagrimal es de 49 años, aunque pueden presentarse a cualquier edad. El $45 \%$ son de origen epitelial (2). En nuestro caso, el diagnóstico anatomopatológico fue de adenocarcinoma. El signo inicial de los tumores malignos de glándula lagrimal es la aparición de una exoftalmía, de evolución relativamente rápida con desplazamiento ocular inferonasal, y se caracterizan por presentar frecuentemente erosión ósea como en el caso de adenocarcinoma que se presenta.

Las lesiones benignas o bien no producen lesiones óseas, o si las producen, consisten en agrandamiento de la fosa lagrimal pero sin signos de osteolisis (3). En este caso se han observado soluciones de continuidad en las paredes óseas que contactaban con el tumor, y que fueron confirmadas posteriormente durante la cirugía. Esto sugería, como así lo confirmo el estudio anatomopatológico posterior, el carácter maligno del tumor. Los tumores malignos de la glándula lagrimal suelen producir además esclerosis ósea y en ocasiones presentar calcifica- ciones, hallazgos encontrados en la TAC realizada a nuestro paciente (3). En contraste con los tumores benignos de la glándula lagrimal, los malignos suelen cursar con exoftalmos doloroso (3). Sin embargo, en nuestro caso, aunque presentaba un exoftalmos acusado, el paciente no refirió haber tenido dolor en ningún momento, probablemente por los escasos signos inflamatorios que presentaba.

Entre los tumores epiteliales malignos de glándula lagrimal se encuentran el carcinoma quístico adenoide, adenocarcinoma, el carcinoma de células escamosas y el carcinoma mucoepidermoide (1). En este caso, el diagnóstico anatomopatológico fue de adenocarcinoma con diferenciación apocrina. El número de pacientes estudiados con adenocarcioma de la glándula lagrimal es reducido debido a que son tumores poco frecuentes. Por esta razón establecer el tratamiento más adecuado es difícil. Un estudio retrospectivo de 13 pacientes (5) concluyó que la exenteración seguida de radioterapia resulta ser el tratamiento más efectivo. En este caso, dado que no había componente inflamatorio evidente y la tumoración estaba bien delimitada, el tratamiento inicial se limitó a la extirpación del tumor. Una vez conocido el diagnóstico anatomopatológico se consultó con el Servicio de Oncología sobre la pauta a seguir. Dadas las patologías concomitantes y el estado general del paciente se decidió que el paciente permaneciese en observación y no se observaron signos de recidiva hasta su fallecimiento por un ACV, un año después de la extirpación del tumor.

\section{BIBLIOGRAFÍA}

1. Alemán Hurtado E, Alemán Blanco S. Neoplasias y quistes orbitarios. In: Alemán Hurtado E, Prat Bartomeu J. Patología Orbitaria: Enfoque multidisciplinar. Madrid: Tecnimedia SL; 1998; 119-123.

2. Shields JA, Shields CL, Scartozzi R. Survey of 1264 patients with orbital tumors and simulating lesions: The 2002 Montgomery Lecture, part 1. Ophthalmology 2004; 111: 997-1008.

3. Char DH. American Cancer Society. Atlas of Clinical Oncology. Tumors of the eye and ocular adnexa. Londres: B C Decker Inc; 2001; 371-385.

4. Shintaku M, Tsuta K, Yoshida H, Tsubura A, Nakashima $Y$, Noda K. Apocrine adenocarcinoma of the eyelid with aggressive biological behaviour: Report of a case. Pathol Int 2002; 52: 169-173.

5. Heaps RS, Miller NR, Albert DM, Green WR, Vitale S. Primary adenocarcinoma of the lacrimal gland. A retrospective study. Ophthalmolgy 1993; 100: 1856-1860. 